\title{
Generalized non-commutative tori
}

\author{
by \\ Chun-Gil PARK (Taejon)
}

\begin{abstract}
The generalized non-commutative torus $T_{\varrho}^{k}$ of rank $n$ is defined by the crossed product $A_{m / k} \times \alpha_{3} \mathbb{Z} \times \alpha_{4} \ldots \times{ }_{\alpha_{n}} \mathbb{Z}$, where the actions $\alpha_{i}$ of $\mathbb{Z}$ on the fibre $M_{k}(\mathbb{C})$ of a rational rotation algebra $A_{m / k}$ are trivial, and $C^{*}(k \mathbb{Z} \times k \mathbb{Z}) \times \alpha_{3} \mathbb{Z} \times \alpha_{\alpha_{4}} \ldots \times{ }_{\alpha_{n}} \mathbb{Z}$ is a non-commutative torus $A_{\varrho}$. It is shown that $T_{\varrho}^{k}$ is strongly Morita equivalent to $A_{\varrho}$, and that $T_{\varrho}^{k} \otimes M_{p}$ is isomorphic to $A_{\varrho} \otimes M_{k}(\mathbb{C}) \otimes M_{p} \infty$ if and only if the set of prime factors of $k$ is a subset of the set of prime factors of $p$.
\end{abstract}

Introduction. Let $G$ be a locally compact abelian group. A multiplier on $G$ is a measurable function $\omega: G \times G \rightarrow \mathbb{T}^{1}$ which satisfies

$$
\begin{aligned}
\omega(x y, z) \omega(x, y) & =\omega(x, y z) \omega(y, z), & & x, y, z \in G, \\
\omega(x, e) & =\omega(e, x)=1, & & x \in G,
\end{aligned}
$$

where $e$ is the identity in $G$. Given a locally compact abelian group $G$ and a multiplier $\omega$ on $G$, one can associate to them the twisted group $C^{*}$-algebra $C^{*}(G, \omega) . C^{*}\left(\mathbb{Z}^{n}, \omega\right)$ is said to be a non-commutative torus of rank $n$ and denoted by $A_{\omega}$. The multiplier $\omega$ determines a subgroup $S_{\omega}$ of $G$, called its symmetry group, and $\omega$ is called totally skew if the symmetry group $S_{\omega}$ is trivial; the torus $A_{\omega}$ is then called completely irrational (see [1]). It was shown in [1] that if $G$ is a locally compact abelian group and $\omega$ is a totally skew multiplier on $G$, then $C^{*}(G, \omega)$ is a simple $C^{*}$-algebra.

Boca [3] showed that almost all completely irrational non-commutative tori are isomorphic to inductive limits of circle algebras, where the "circle algebra" means a $C^{*}$-algebra which is a finite direct sum of $C^{*}$-algebras of the form $C\left(\mathbb{T}^{1}\right) \otimes M_{q}(\mathbb{C})$. We will assume that each completely irrational non-commutative torus appearing in this paper is an inductive limit of circle algebras.

2000 Mathematics Subject Classification: Primary 46L05, 46L87.

Key words and phrases: non-commutative torus, equivalence bimodule, circle algebra, real rank 0, crossed product, UHF-algebra, Cuntz algebra.

Research supported by grant No. 1999-2-102-001-3 from the interdisciplinary Research program year of the KOSEF. The author would also like to thank the referee for a number of valuable suggestions to a previous version of this paper. 
In [5], it was shown that two separable $C^{*}$-algebras $A$ and $B$ are stably isomorphic if and only if they are strongly Morita equivalent, i.e., there exists an $A$ - $B$-equivalence bimodule defined in [14]. In [4], M. Brabanter constructed an $A_{m / k}-C\left(\mathbb{T}^{2}\right)$-equivalence bimodule. Modifying his construction, we are going to construct a $T_{\varrho}^{k}$ - $A_{\varrho}$-equivalence bimodule.

It was shown in [2, Theorem 1.5] that each completely irrational noncommutative torus has real rank 0 , where the "real rank 0" means that the set of invertible self-adjoint elements is dense in the set of self-adjoint elements. Combining Theorem 1.2 given in the first section and [6, Corollary $3.3]$ yields that if $A_{\varrho}$ is simple then $T_{\varrho}^{k}$ has real rank 0 , since the noncommutative torus $A_{\varrho}$ has real rank 0. And Lin and Rørdam's results [12, Propositions 2 and 3] say that if $A_{\varrho}$ simple then $T_{\varrho}^{k}$ is an inductive limit of circle algebras, since $T_{\varrho}^{k} \otimes \mathcal{K}(\mathcal{H}) \cong A_{\varrho} \otimes \mathcal{K}(\mathcal{H})$ is an inductive limit of circle algebras.

Combining Elliott's classification theorem [10, Theorem 7.1] and Ji and Xia's result [11, Theorem 1.3] yields that the completely irrational noncommutative tori $A_{\omega}$ of rank $n$ and the simple generalized non-commutative tori $T_{\varrho}^{k}$ of rank $n$ are classified by the ranges of the traces, and so one can completely classify them up to isomorphism or up to strong Morita equivalence. Hence some completely irrational non-commutative tori $A_{\omega}$ of rank $n$ are isomorphic to some simple generalized non-commutative tori $T_{\varrho}^{k}$ of rank $n$, and this result can be applied to understand the (bundle) structure of $C^{*}$-algebras of sections of locally trivial continuous $C^{*}$-algebra bundles over $\mathrm{CW}$-complexes with fibres completely irrational non-commutative tori.

It is moreover shown that $T_{\varrho}^{k} \otimes M_{p^{\infty}}$ is isomorphic to $A_{\varrho} \otimes M_{k}(\mathbb{C}) \otimes M_{p^{\infty}}$ if and only if the set of prime factors of $k$ is a subset of the set of prime factors of $p$, that $\mathcal{O}_{2 u} \otimes T_{\varrho}^{k}$ is isomorphic to $\mathcal{O}_{2 u} \otimes A_{\varrho} \otimes M_{k}(\mathbb{C})$ if and only if $k$ and $2 u-1$ are relatively prime, and that $\mathcal{O}_{\infty} \otimes T_{\varrho}^{k}$ is not isomorphic to $\mathcal{O}_{\infty} \otimes A_{\varrho} \otimes M_{k}(\mathbb{C})$ if $k>1$, where $\mathcal{O}_{u}$ and $\mathcal{O}_{\infty}$ denote the Cuntz algebra and the generalized Cuntz algebra, respectively.

1. Generalized non-commutative tori. It was shown in [4, Proposition 1] that $A_{m / k}$ is the $C^{*}$-algebra of matrices $\left(f_{i j}\right)_{i, j=1}^{k}$ of functions $f_{i j}$ with

$$
\begin{array}{ll}
f_{i j} \in C^{*}(k \mathbb{Z} \times k \mathbb{Z}) & \text { if } i, j \in\{1, \ldots, k-1\} \text { or }(i, j)=(k, k), \\
f_{i k} \in \Omega \& f_{k i} \in \Omega^{*} & \text { if } i \in\{1, \ldots, k-1\},
\end{array}
$$

where $\Omega$ and $\Omega^{*}$ are the $C^{*}(k \mathbb{Z} \times k \mathbb{Z})$-modules defined as

$$
\begin{aligned}
\Omega & =\left\{f \in C(\widehat{k \mathbb{Z}} \times[0,1]) \mid f(z, 1)=z^{s} f(z, 0), \forall z \in \widehat{k \mathbb{Z}}\right\}, \\
\Omega^{*} & =\left\{f \in C(\widehat{k \mathbb{Z}} \times[0,1]) \mid f^{*} \in \Omega\right\}
\end{aligned}
$$

for an integer $s$ such that $s m=1(\bmod k)$. 
The non-commutative torus $A_{\omega}$ of rank $n$ is obtained by an iteration of $n-1$ crossed products by actions of $\mathbb{Z}$, the first action being on $C\left(\mathbb{T}^{1}\right)$. When $A_{\omega}$ has a primitive ideal space $\widehat{S}_{\omega} \cong \widehat{k \mathbb{Z}}, A_{\omega}$ is realized as the $C^{*}$-algebra of sections of a locally trivial continuous $C^{*}$-algebra bundle over $\widehat{k \mathbb{Z}}$ with fibres $C^{*}\left(\mathbb{Z}^{n} / S_{\omega}, \omega_{1}\right)$ for some totally skew multiplier $\omega_{1}$, where $C^{*}\left(\mathbb{Z}^{n} / S_{\omega}, \omega_{1}\right) \cong$ $A_{\varphi} \otimes M_{k}(\mathbb{C})$ for $A_{\varphi}$ a completely irrational non-commutative torus of rank $n-1$. By a change of basis, one can assume that $A_{\omega} \cong A_{m / k} \times \alpha_{3} \mathbb{Z} \times \alpha_{4}$ $\ldots \times_{\alpha_{n}} \mathbb{Z}$, where the actions $\alpha_{i}$ of $\mathbb{Z}$ on the fibre $M_{k}(\mathbb{C})$ of $A_{m / k}$ are trivial, since the fibre $M_{k}(\mathbb{C})$ of $A_{m / k}$ is factored out of the fibre $C^{*}\left(\mathbb{Z}^{n} / S_{\omega}, \omega_{1}\right)$ of $A_{\omega}$ (see $[1,9,13]$ ). This assures us of the existence of such actions $\alpha_{i}$ as in the definition of $T_{\varrho}^{k}$ in the abstract.

1.1. Definition. The generalized non-commutative torus $T_{\varrho}^{k}$ of rank $n$ is defined to be the crossed product $A_{m / k} \times{ }_{\alpha_{3}} \mathbb{Z} \times_{\alpha_{4}} \ldots \times_{\alpha_{n}} \mathbb{Z}$, where the actions $\alpha_{i}$ of $\mathbb{Z}$ on the fibre $M_{k}(\mathbb{C})$ of a rational rotation algebra $A_{m / k}$ are trivial, and $C^{*}(k \mathbb{Z} \times k \mathbb{Z}) \times_{\alpha_{3}} \mathbb{Z} \times_{\alpha_{4}} \ldots \times_{\alpha_{n}} \mathbb{Z}$ is a non-commutative torus $A_{\varrho}$ of rank $n$.

So the generalized non-commutative torus $T_{\varrho}^{k}$ has a matrix representation induced from the matrix representation of the rational rotation subalgebra $A_{m / k}$.

1.2. Proposition. The generalized non-commutative torus $T_{\varrho}^{k}$ is isomorphic to the $C^{*}$-algebra of matrices $\left(g_{i j}\right)_{i, j=1}^{k}$ with

$$
\begin{array}{ll}
g_{i j} \in A_{\varrho} & \text { if } i, j \in\{1, \ldots, k-1\} \text { or }(i, j)=(k, k), \\
g_{i k} \in \widetilde{\Omega} \& g_{k i} \in \widetilde{\Omega}^{*} & \text { if } i \in\{1, \ldots, k-1\},
\end{array}
$$

where $\widetilde{\Omega}$ and $\widetilde{\Omega}^{*}$ are the $A_{\varrho}$-modules defined as

$$
\widetilde{\Omega}=A_{\varrho} \cdot \Omega, \quad \widetilde{\Omega}^{*}=A_{\varrho} \cdot \Omega^{*} .
$$

Here $\Omega$ and $\Omega^{*}$ are the $C^{*}(k \mathbb{Z} \times k \mathbb{Z})$-modules defined above.

Proof. One sees from the definition of $T_{\varrho}^{k}$ that the isomorphism between $A_{m / k}$ and the $C^{*}$-algebra of matrices $\left(f_{i j}\right)_{i, j=1}^{k}$ satisfying the condition given above gives an isomorphism between $T_{\varrho}^{k}$ and the $C^{*}$-algebra of matrices $\left(g_{i j}\right)_{i, j=1}^{k}$ satisfying the condition given in the statement. Note that $\widetilde{\Omega}$ and $\widetilde{\Omega}^{*}$ are the $A_{\varrho}$-modules defined by canonically replacing $C^{*}(k \mathbb{Z} \times k \mathbb{Z})$ in $\Omega=C^{*}(k \mathbb{Z} \times k \mathbb{Z}) \cdot \Omega$ with $A_{\varrho} \cong C^{*}(k \mathbb{Z} \times k \mathbb{Z}) \times_{\alpha_{3}} \mathbb{Z} \times_{\alpha_{4}} \ldots \times_{\alpha_{n}} \mathbb{Z}$, since the entries in the matrix representation of $A_{m / k}$ have a $C^{*}(k \mathbb{Z} \times k \mathbb{Z})$-module structure, and $T_{\varrho}^{k}$ may be obtained by canonically replacing $C^{*}(k \mathbb{Z} \times k \mathbb{Z})$ with $A_{\varrho} \cong C^{*}(k \mathbb{Z} \times k \mathbb{Z}) \times_{\alpha_{3}} \mathbb{Z} \times_{\alpha_{4}} \ldots \times_{\alpha_{n}} \mathbb{Z}$.

We are going to construct a $T_{\varrho}^{k}-A_{\varrho}$-equivalence bimodule. 
1.3. Theorem. $T_{\varrho}^{k}$ is strongly Morita equivalent to $A_{\varrho}$.

Proof. Let $X$ be the complex vector space $\left(\bigoplus_{1}^{k-1} \widetilde{\Omega}\right) \oplus A_{\varrho}$. We will consider the elements of $X$ as $(k, 1)$ matrices where the first $k-1$ entries are in $\widetilde{\Omega}$ and the last entry is in $A_{\varrho}$. If $x \in X$, denote by $x^{*}$ the $(1, k)$ matrix resulting from $x$ by transposition and involution so that $x^{*} \in\left(\bigoplus_{1}^{k-1} \widetilde{\Omega}^{*}\right) \oplus$ $A_{\varrho}$. The space $X$ is a left $T_{\varrho}^{k}$-module if module multiplication is defined by matrix multiplication $F \cdot x$, where $F=\left(g_{i j}\right)_{i, j=1}^{k} \in T_{\varrho}^{k}$ and $x \in X$. If $g \in A_{\varrho}$ and $x \in X$, then $x \cdot[g]$ defines a right $A_{\varrho}$-module structure on $X$. Now we define a $T_{\varrho}^{k}$-valued and an $A_{\varrho}$-valued inner products $\langle\cdot, \cdot\rangle_{T_{\varrho}^{k}}$ and $\langle\cdot, \cdot\rangle_{A_{\varrho}}$ on $X$ by

$$
\langle x, y\rangle_{T_{e}^{k}}=x \cdot y^{*}, \quad\langle x, y\rangle_{A_{\varrho}}=x^{*} \cdot y
$$

for $x, y \in X$, with matrix multiplication on the right.

It is obvious that for $x, y \in X, x \cdot y^{*} \in T_{\varrho}^{k}$ and $x^{*} \cdot y \in A_{\varrho}$. Let $A_{m / k}=T_{\varrho}^{k}$. By [4, Theorem 3], $\left\{x \cdot y^{*} \mid x, y \in X\right\}$ is dense in $A_{m / k}$. Let us replace $C^{*}(k \mathbb{Z} \times k \mathbb{Z})$ in the vector space $X$ for $A_{m / k}$ with $A_{\varrho} \cong C^{*}(k \mathbb{Z} \times k \mathbb{Z}) \times_{\alpha_{3}}$ $\mathbb{Z} \times_{\alpha_{4}} \ldots \times_{\alpha_{n}} \mathbb{Z}$. From the definitions of $\widetilde{\Omega}$ and $\widetilde{\Omega}^{*}$ and the structure of the generalized non-commutative torus $T_{\varrho}^{k}$ of rank $n$, given in the proof of Proposition 1.2, one finds that $\left\{x \cdot y^{*} \mid x, y \in X\right\}$ is dense in $T_{\varrho}^{k}$. On the other hand, for any $a \in A_{\varrho}$, let $x=(0,0, \ldots, 0,1), y=(0,0, \ldots, 0, a) \in X$. Then $x^{*} \cdot y=a$. Hence $\left\{x^{*} \cdot y \mid x, y \in X\right\}$ is dense in $A_{\varrho}$. So $X$ becomes a $T_{\varrho}^{k}$ - $A_{\varrho}$-equivalence bimodule, as desired.

The generalized non-commutative torus $T_{\varrho}^{k}$ of rank $n$ is strongly Morita equivalent to the non-commutative torus $A_{\varrho}$ of rank $n$, so $K_{i}\left(T_{\varrho}^{k}\right) \cong K_{i}\left(A_{\varrho}\right)$ $\cong \mathbb{Z}^{2^{n-1}}$ (see [9, Theorem 2.2]). The non-commutative torus $A_{\varrho}$ of rank $n$ is the universal object for unitary $\varrho$-representations of $\mathbb{Z}^{n}$, so $A_{\varrho}$ is realized as $C^{*}\left(u_{1}, \ldots, u_{n} \mid u_{i} u_{j}=e^{2 \pi i \theta_{j i}} u_{j} u_{i}\right)$, where $u_{i}$ are unitaries and $\theta_{j i}$ are real numbers for $1 \leq i, j \leq n$.

1.4. Theorem. (1) $\operatorname{tr}\left(K_{0}\left(T_{\varrho}^{k}\right)\right)=k^{-1} \cdot \operatorname{tr}\left(K_{0}\left(A_{\varrho}\right)\right)$ if $A_{\varrho}$ is completely irrational.

(2) $\left[1_{T_{\varrho}^{k}}\right] \in K_{0}\left(T_{\varrho}^{k}\right)$ is primitive.

Proof. (1) $T_{\varrho}^{k}$ has a matrix representation induced from the matrix representation of the rational rotation subalgebra $A_{m / k}$. The diagonal entries of the matrix representation are in $A_{\varrho}$, and so the range of the trace of $K_{0}\left(T_{\varrho}^{k}\right)$ is

$$
\mathbb{Z}+\frac{1}{k}(\mathbb{Z}+\mathbb{Z} \alpha+\mathbb{Z} \beta+\ldots+\mathbb{Z} \gamma)
$$

where $\operatorname{tr}\left(K_{0}\left(A_{\varrho}\right)\right)=\mathbb{Z}+\mathbb{Z} k+\mathbb{Z} \alpha+\mathbb{Z} \beta+\ldots+\mathbb{Z} \gamma$. Hence $\operatorname{tr}\left(K_{0}\left(T_{\varrho}^{k}\right)\right)=$ $k^{-1} \cdot \operatorname{tr}\left(K_{0}\left(A_{\varrho}\right)\right)$. 
(2) We argue by induction on $n$. For $n=2$, it is the Elliott result [9, Theorem 2.2]. Assume that the result is true for all $T_{\varrho}^{k}$ with $n=i-1$. Since $T_{\varrho}^{k}$ is realized as $A_{m / k} \times \alpha_{\alpha_{3}} \mathbb{Z} \times{ }_{\alpha_{4}} \ldots \times_{\alpha_{n}} \mathbb{Z}$, write $\mathbb{S}_{i}=C^{*}\left(\mathbb{S}_{i-1}, u_{i}\right)$, where $\mathbb{S}_{i}=C^{*}\left(A_{m / k}, u_{3}, \ldots, u_{i}\right)$. Then the inductive hypothesis applies to $\mathbb{S}_{i-1}$. Also, we can think of $\mathbb{S}_{i}$ as the crossed product of $\mathbb{S}_{i-1}$ by an action $\alpha_{i}$ of $\mathbb{Z}$, where the generator of $\mathbb{Z}$ corresponds to $u_{i}$, which acts on $C^{*}\left(u_{1}^{k}, u_{2}^{k}, u_{3}, \ldots, u_{i-1}\right)$ by conjugation (sending $u_{j}$ to $u_{i} u_{j} u_{i}^{-1}=e^{2 \pi i \theta_{j i}} u_{j}$, $j \neq 1,2$, and sending $u_{j}^{k}$ to $\left.u_{i} u_{j}^{k} u_{i}^{-1}=e^{2 \pi i k \theta_{j i}} u_{j}^{k}, j=1,2\right)$, and which acts trivially on $M_{k}(\mathbb{C})$. Note that this action is homotopic to the trivial action, since we can homotope $\theta_{j i}$ to 0 . Hence $\mathbb{Z}$ acts trivially on the $K$-theory of $\mathbb{S}_{i-1}$. The Pimsner-Voiculescu exact sequence for a crossed product gives an exact sequence

$$
K_{0}\left(\mathbb{S}_{i-1}\right) \stackrel{1-\left(\alpha_{i}\right)_{*}}{\longrightarrow} K_{0}\left(\mathbb{S}_{i-1}\right) \stackrel{\Phi}{\rightarrow} K_{0}\left(\mathbb{S}_{i}\right) \rightarrow K_{1}\left(\mathbb{S}_{i-1}\right) \stackrel{1-\left(\alpha_{i}\right)_{*}}{\longrightarrow} K_{1}\left(\mathbb{S}_{i-1}\right)
$$

and similarly for $K_{1}$, where the map $\Phi$ is induced by inclusion. Since $\left(\alpha_{i}\right)_{*}=$ 1 and since the $K$-groups of $\mathbb{S}_{i-1}$ are free abelian, this reduces to a split short exact sequence

$$
\{0\} \rightarrow K_{0}\left(\mathbb{S}_{i-1}\right) \stackrel{\Phi}{\rightarrow} K_{0}\left(\mathbb{S}_{i}\right) \rightarrow K_{1}\left(\mathbb{S}_{i-1}\right) \rightarrow\{0\}
$$

and similarly for $K_{1}$. So $K_{0}\left(\mathbb{S}_{i}\right)$ and $K_{1}\left(\mathbb{S}_{i}\right)$ are free abelian of rank $2 \cdot 2^{i-2}$ $=2^{i-1}$. Furthermore, since the inclusion $\mathbb{S}_{i-1} \rightarrow \mathbb{S}_{i}$ sends $1_{\mathbb{S}_{i-1}}$ to $1_{\mathbb{S}_{i}},\left[1_{\mathbb{S}_{i}}\right]$ is the image of $\left[1_{\mathbb{S}_{i-1}}\right]$, which is primitive in $K_{0}\left(\mathbb{S}_{i-1}\right)$ by inductive hypothesis. Hence the image is primitive, since the Pimsner-Voiculescu exact sequence is a split short exact sequence of torsion-free groups.

1.5. Corollary. $T_{\varrho}^{k}$ is not isomorphic to $A \otimes M_{d}(\mathbb{C})$ for a $C^{*}$-algebra $A$ if $d>1$.

Proof. Assume $T_{\varrho}^{k}$ is isomorphic to $A \otimes M_{d}(\mathbb{C})$. Then the unit $1_{T_{\varrho}^{k}}$ maps to $1_{A} \otimes I_{d}$. This implies that there is a projection $e$ in $T_{\varrho}^{k}$ such that $\left[1_{T_{\varrho}^{k}}\right]=$ $d[e]$ in $K_{0}\left(T_{\varrho}^{k}\right)$, which contradicts Theorem 1.4 if $d>1$. Thus no non-trivial matrix algebra can be factored out of $T_{\varrho}^{k}$.

2. Tensor products of generalized non-commutative tori with UHF-algebras and Cuntz algebras. Using the fact that $\left[1_{T_{\varrho}^{k}}\right] \in K_{0}\left(T_{\varrho}^{k}\right)$ is primitive, we investigate the structure of $T_{\varrho}^{k} \otimes M_{p^{\infty}}$ for $M_{p^{\infty}}$ a UHFalgebra of type $p^{\infty}$.

2.1. Theorem. $T_{\varrho}^{k} \otimes M_{p \infty}$ is isomorphic to $A_{\varrho} \otimes M_{k}(\mathbb{C}) \otimes M_{p \infty}$ if and only if the set of prime factors of $k$ is a subset of the set of prime factors of $p$.

Proof. Assume that the set of prime factors of $k$ is a subset of the set of prime factors of $p$. To show that $T_{\varrho}^{k} \otimes M_{p^{\infty}}$ is isomorphic to $A_{\varrho} \otimes M_{k}(\mathbb{C}) \otimes$ 
$M_{p^{\infty}}$, it is enough to show that $T_{\varrho}^{k} \otimes M_{k^{\infty}} \cong A_{\varrho} \otimes M_{k}(\mathbb{C}) \otimes M_{k^{\infty}}$. But there exist the $C^{*}$-algebra homomorphisms which are the canonical inclusions $T_{\varrho}^{k} \otimes M_{k^{g}}(\mathbb{C}) \hookrightarrow A_{\varrho} \otimes M_{k}(\mathbb{C}) \otimes M_{k^{g}}(\mathbb{C})$ and the $A_{\varrho^{-}}$-module maps $A_{\varrho} \otimes$ $M_{k^{g}}(\mathbb{C}) \hookrightarrow T_{\varrho}^{k} \otimes M_{k^{g}}(\mathbb{C}):$

$$
T_{\varrho}^{k} \hookrightarrow A_{\varrho} \otimes M_{k}(\mathbb{C}) \hookrightarrow T_{\varrho}^{k} \otimes M_{k}(\mathbb{C}) \hookrightarrow A_{\varrho} \otimes M_{k^{2}}(\mathbb{C}) \hookrightarrow \ldots
$$

The inductive limit of the odd terms

$$
\ldots \rightarrow T_{\varrho}^{k} \otimes M_{k^{g}}(\mathbb{C}) \rightarrow T_{\varrho}^{k} \otimes M_{k^{g+1}}(\mathbb{C}) \rightarrow \ldots
$$

is $T_{\varrho}^{k} \otimes M_{k}$, and the inductive limit of the even terms

$$
\ldots \rightarrow A_{\varrho} \otimes M_{k^{g}}(\mathbb{C}) \rightarrow A_{\varrho} \otimes M_{k^{g+1}}(\mathbb{C}) \rightarrow \ldots
$$

is $A_{\varrho} \otimes M_{k^{\infty}}$. Thus by the Elliott theorem [10, Theorem 2.1], $T_{\varrho}^{k} \otimes M_{k^{\infty}}$ is isomorphic to $A_{\varrho} \otimes M_{k \infty}$.

Conversely, assume that $T_{\varrho}^{k} \otimes M_{p^{\infty}} \cong A_{\varrho} \otimes M_{k}(\mathbb{C}) \otimes M_{p^{\infty}}$. Then the unit $1_{T_{\varrho}^{k}} \otimes 1_{M_{p} \infty}$ maps to the unit $1_{A_{\varrho}} \otimes 1_{M_{p} \infty} \otimes I_{k}$. So

$$
\begin{aligned}
{\left[1_{T_{\varrho}^{k}} \otimes 1_{M_{p} \infty}\right] } & =\left[1_{A_{\varrho}} \otimes 1_{M_{p} \infty} \otimes I_{k}\right], \\
{\left[1_{T_{\varrho}^{k}} \otimes 1_{M_{p} \infty}\right] } & =\left[1_{T_{\varrho}^{k}}\right] \otimes\left[1_{M_{p} \infty}\right], \\
{\left[1_{A_{\varrho}} \otimes 1_{M_{p} \infty} \otimes I_{k}\right] } & =k\left(\left[1_{A_{\varrho}}\right] \otimes\left[1_{M_{p} \infty}\right]\right) .
\end{aligned}
$$

Under the assumption that the unit $1_{T_{o}^{k}} \otimes 1_{M_{p} \infty}$ maps to the unit $1_{A_{\varrho}} \otimes$ $1_{M_{p} \infty} \otimes I_{k}$, if there is a prime factor $q$ of $k$ such that $q \nmid p$, then $\left[1_{M_{p} \infty}\right] \neq q\left[e_{\infty}\right]$ for $e_{\infty}$ a projection in $M_{p^{\infty}}$. So there is a projection $e \in T_{\varrho}^{k}$ such that $\left[1_{T_{e}^{k}}\right]=q[e]$. This contradicts Theorem 1.4. Thus the set of prime factors of $k$ is a subset of the set of prime factors of $p$.

Therefore, $T_{\varrho}^{k} \otimes M_{p^{\infty}}$ is isomorphic to $A_{\varrho} \otimes M_{k}(\mathbb{C}) \otimes M_{p^{\infty}}$ if and only if the set of prime factors of $k$ is a subset of the set of prime factors of $p$.

Let us study the structure of the tensor products of generalized noncommutative tori with (even) Cuntz algebras.

The Cuntz algebra $\mathcal{O}_{u}, 2 \leq u<\infty$, is the universal $C^{*}$-algebra generated by $u$ isometries $s_{1}, \ldots, s_{u}$, i.e., $s_{j}^{*} s_{j}=1$ for all $j$, with the relation $s_{1} s_{1}^{*}+$ $\ldots+s_{u} s_{u}^{*}=1$. Cuntz $[7,8]$ proved that $\mathcal{O}_{u}$ is simple and the $K$-theory of $\mathcal{O}_{u}$ is $K_{0}\left(\mathcal{O}_{u}\right)=\mathbb{Z} /(u-1) \mathbb{Z}$ and $K_{1}\left(\mathcal{O}_{u}\right)=0$. He proved that $K_{0}\left(\mathcal{O}_{u}\right)$ is generated by the class of the unit.

2.2. Proposition. Let $u$ be a positive integer such that $k$ and $u-1$ are not relatively prime. Then $\mathcal{O}_{u} \otimes T_{\varrho}^{k}$ is not isomorphic to $\mathcal{O}_{u} \otimes A_{\varrho} \otimes M_{k}(\mathbb{C})$.

Proof. Let $p$ be a prime such that $p \mid k$ and $p \mid u-1$. Suppose that $\mathcal{O}_{u} \otimes T_{\varrho}^{k}$ is isomorphic to $\mathcal{O}_{u} \otimes A_{\varrho} \otimes M_{k}(\mathbb{C})$. Then the unit $1_{\mathcal{O}_{u} \otimes T_{\varrho}^{k}}$ maps to the unit $1_{\mathcal{O}_{u} \otimes A_{\varrho}} \otimes I_{k}$. So $\left[1_{\mathcal{O}_{u} \otimes T_{\varrho}^{k}}\right]=\left[1_{\mathcal{O}_{u} \otimes A_{\varrho}} \otimes I_{k}\right]=k\left[1_{\mathcal{O}_{u} \otimes A_{\varrho}}\right]$. Hence there is a projection $e$ in $\mathcal{O}_{u} \otimes T_{\varrho}^{k}$ such that $\left[1_{\mathcal{O}_{u} \otimes T_{\varrho}^{k}}\right]=k[e]$. But $\left[1_{\mathcal{O}_{u} \otimes T_{\varrho}^{k}}\right]=$ 
$\left[1_{\mathcal{O}_{u}}\right] \otimes\left[1_{T_{e}^{k}}\right]$ and $\left[1_{\mathcal{O}_{u}}\right]$ is a generator of $K_{0}\left(\mathcal{O}_{u}\right) \cong \mathbb{Z} /(u-1) \mathbb{Z}$ (see [8]). But $p \mid u-1$. We have $\left[1_{\mathcal{O}_{u}}\right] \neq p\left[e_{*}\right]$ for $e_{*}$ a projection in $\mathcal{O}_{u}$. So $\left[1_{T_{e}^{k}}\right]=p\left[e^{\prime}\right]$ for $e^{\prime}$ a projection in $T_{\varrho}^{k}$. This contradicts Theorem 1.4. Hence $k$ and $u-1$ are relatively prime.

Therefore, $\mathcal{O}_{u} \otimes T_{\varrho}^{k}$ is not isomorphic to $\mathcal{O}_{u} \otimes A_{\varrho} \otimes M_{k}(\mathbb{C})$ if $k$ and $u-1$ are not relatively prime.

The following result is useful to understand the structure of $\mathcal{O}_{u} \otimes T_{\varrho}^{k}$.

2.3. Proposition [15, Theorem 7.2]. Let $A$ and $B$ be unital simple inductive limits of even Cuntz algebras. If $\alpha: K_{0}(A) \rightarrow K_{0}(B)$ is an isomorphism of abelian groups satisfying $\alpha\left(\left[1_{A}\right]\right)=\left[1_{B}\right]$, then there is an isomorphism $\phi: A \rightarrow B$ which induces $\alpha$.

2.4. Corollary. (1) Let $p$ be an odd integer such that $p$ and $2 u-1$ are relatively prime. Then $\mathcal{O}_{2 u}$ is isomorphic to $\mathcal{O}_{(2 u-1) p+1} \otimes M_{p^{\infty}}$. That is, $\mathcal{O}_{2 u}$ is isomorphic to $\mathcal{O}_{2 u} \otimes M_{p^{\infty}}$.

(2) $\mathcal{O}_{2 u}$ is isomorphic to $\mathcal{O}_{2 u} \otimes M_{(2 u)^{\infty}}$.

2.5. TheOREM. $\mathcal{O}_{2 u} \otimes T_{\varrho}^{k}$ is isomorphic to $\mathcal{O}_{2 u} \otimes A_{\varrho} \otimes M_{k}(\mathbb{C})$ if and only if $k$ and $2 u-1$ are relatively prime.

Proof. Assume that $k$ and $2 u-1$ are relatively prime. Let $k=p 2^{v}$ for some odd integer $p$. Then $p$ and $2 u-1$ are relatively prime. Then by Corollary 2.4, $\mathcal{O}_{2 u}$ is isomorphic to $\mathcal{O}_{2 u} \otimes M_{p^{\infty}}$, and $\mathcal{O}_{2 u}$ is isomorphic to $\mathcal{O}_{2 u} \otimes M_{(2 u)^{\infty}} \cong \mathcal{O}_{2 u} \otimes M_{(2 u)^{\infty}} \otimes M_{\left(2^{v}\right)^{\infty}} \cong \mathcal{O}_{2 u} \otimes M_{\left(2^{v}\right)^{\infty}}$. So $\mathcal{O}_{2 u}$ is isomorphic to $\mathcal{O}_{2 u} \otimes M_{p^{\infty}} \otimes M_{\left(2^{v}\right)^{\infty}} \cong \mathcal{O}_{2 u} \otimes M_{k^{\infty}}$. Thus by Theorem 2.1, $\mathcal{O}_{2 u} \otimes T_{\varrho}^{k}$ is isomorphic to $\mathcal{O}_{2 u} \otimes M_{k^{\infty}} \otimes T_{\varrho}^{k}$, which in turn is isomorphic to $\mathcal{O}_{2 u} \otimes M_{k} \otimes A_{\varrho} \otimes M_{k}(\mathbb{C})$. Hence $\mathcal{O}_{2 u} \otimes T_{\varrho}^{k}$ is isomorphic to $\mathcal{O}_{2 u} \otimes A_{\varrho} \otimes M_{k}(\mathbb{C})$.

The converse was proved in Proposition 2.2.

Therefore, $\mathcal{O}_{2 u} \otimes T_{\varrho}^{k}$ is isomorphic to $\mathcal{O}_{2 u} \otimes A_{\varrho} \otimes M_{k}(\mathbb{C})$ if and only if $k$ and $2 u-1$ are relatively prime.

Cuntz [8] computed the $K$-theory of the generalized Cuntz algebra $\mathcal{O}_{\infty}$, generated by a sequence of isometries with mutually orthogonal ranges, $K_{0}\left(\mathcal{O}_{\infty}\right)=\mathbb{Z}$ and $K_{1}\left(\mathcal{O}_{\infty}\right)=0$. He proved that $K_{0}\left(\mathcal{O}_{\infty}\right)$ is generated by the class of the unit.

2.6. Proposition. $\mathcal{O}_{\infty} \otimes T_{\varrho}^{k}$ is not isomorphic to $\mathcal{O}_{\infty} \otimes A_{\varrho} \otimes M_{k}(\mathbb{C})$ if $k>1$.

Proof. Suppose $\mathcal{O}_{\infty} \otimes T_{\varrho}^{k}$ is isomorphic to $\mathcal{O}_{\infty} \otimes A_{\varrho} \otimes M_{k}(\mathbb{C})$. The unit $1_{\mathcal{O}_{\infty} \otimes T_{\varrho}^{k}}$ maps to the unit $1_{\mathcal{O}_{\infty} \otimes A_{\varrho}} \otimes I_{k}$. By the same trick as in the proof of Proposition 2.2, one can show that $\left[1_{\mathcal{O}_{\infty} \otimes T_{\varrho}^{k}}\right]=k[e]$ for a projection $e \in \mathcal{O}_{\infty} \otimes T_{\varrho}^{k}$. We have $\left[1_{\mathcal{O}_{\infty} \otimes T_{\varrho}^{k}}\right]=\left[1_{\mathcal{O}_{\infty}}\right] \otimes\left[1_{T_{\varrho}^{k}}\right]$ and $\left[1_{\mathcal{O}_{\infty}}\right]$ is a primitive 
element of $K_{0}\left(\mathcal{O}_{\infty}\right) \cong \mathbb{Z}$ (see [8]). So $\left[1_{T_{\varrho}^{k}}\right]=k\left[e^{\prime}\right]$ for a projection $e^{\prime} \in T_{\varrho}^{k}$. This contradicts Theorem 1.4 if $k>1$.

Therefore, $\mathcal{O}_{\infty} \otimes T_{\varrho}^{k}$ is not isomorphic to $\mathcal{O}_{\infty} \otimes A_{\varrho} \otimes M_{k}(\mathbb{C})$.

\section{References}

[1] L. Baggett and A. Kleppner, Multiplier representations of abelian groups, J. Funct. Anal. 14 (1973), 299-324.

[2] B. Blackadar, A. Kumjian and M. Rørdam, Approximately central matrix units and the structure of non-commutative tori, K-Theory 6 (1992), 267-284.

[3] F. Boca, The structure of higher-dimensional non-commutative tori and metric Diophantine approximation, J. Reine Angew. Math. 492 (1997), 179-219.

[4] M. Brabanter, The classification of rational rotation $C^{*}$-algebras, Arch. Math. (Basel) 43 (1984), 79-83.

[5] L. Brown, P. Green and M. Rieffel, Stable isomorphism and strong Morita equivalence of $C^{*}$-algebras, Pacific J. Math. 71 (1977), 349-363.

[6] L. Brown and G. Pedersen, $C^{*}$-algebras of real rank zero, J. Funct. Anal. 99 (1991), 131-149.

[7] J. Cuntz, Simple $C^{*}$-algebras generated by isometries, Comm. Math. Phys. 57 (1977), 173-185.

[8] -, K-theory for certain $C^{*}$-algebras, Ann. of Math. 113 (1981), 181-197.

[9] G. Elliott, On the $K$-theory of the $C^{*}$-algebra generated by a projective representation of a torsion-free discrete abelian group, in: Operator Algebras and Group Representations, Vol. 1, G. Aresene et al. (eds.), Pitman, London, 1984, 157-184.

[10] - On the classification of $C^{*}$-algebras of real rank zero, J. Reine Angew. Math. 443 (1993), 179-219.

[11] R. Ji and J. Xia, On the classification of commutator ideals, J. Funct. Anal. 78 (1988), 208-232.

[12] H. Lin and M. Rørdam, Extensions of inductive limits of circle algebras, J. London Math. Soc. 51 (1995), 603-613.

[13] S. Oh and C. Park, The bundle structure of non-commutative tori, Vietnam J. Math. 27 (1999), 301-308.

[14] M. Rieffel, Morita equivalence for operator algebras, in: Operator Algebras and Applications, R. V. Kadison (ed.), Proc. Sympos. Pure Math. 38, Amer. Math. Soc., Providence, RI, 1982, 285-298.

[15] M. Rørdam, Classification of inductive limits of Cuntz algebras, J. Reine Angew. Math. 440 (1993), 175-200.

Department of Mathematics

Chungnam National University

Taejon 305-764, South Korea

E-mail: cgpark@math.chungnam.ac.kr

Received March 13, 2000

Revised version August 20, 2001 